Teubner Studienbücher Physik

Johann Bienlein, Roland Wiesendanger

\author{
Einführung in \\ die Stuktur der Materie
}


Johann Bienlein, Roland Wiesendanger

\section{Einführung in \\ die Struktur der Materie}

\section{Kerne, Teilchen, Moleküle, Festkörper}

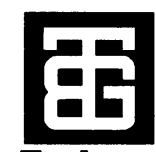

Teubner

B. G. Teubner Stuttgart - Leipzig - Wiesbaden 
Bibliografische Information der Deutschen Bibliothek

Die Deutsche Bibliothek verzeichnet diese Publikation in der Deutschen Nationalbibliographie; detaillierte bibliografische Daten sind im Internet über <http://dnb.ddb.de> abrufbar.

\section{Prof. Dr. Johann Konrad Bienlein}

Geboren 1930 in Bayreuth, Schule in Coburg, Studium und Promotion (1958) in Erlangen bei Prof. Dr. Fleischmann, dort auch Wissenschaftlicher Assistent und Habilitation.

Weitere wissenschaftliche Tätigkeiten am Oak Ridge National Laboratory/TN/USA und bei CERN/Genf, 1967 Berufung als Leitender Wissenschaftler Zu DESY/Hamburg, 1971 Professor an der Universität Hamburg, Gastaufenthalte bei CERN/Genf, Stanford/CANSA und der ETH-Zürich/Schweiz. 1998 Honorarprofessor am Henryk Niewodniczanski Institut für Kernphysik in Krakau/Polen

\section{Prof. Dr. Roland Wiesendanger}

Geboren 1961 in Basel, Schweiz. Physikstudium, Promotion und Habilitation in Experimenteller Physik an der Universität Basel. Seit 1993 Professor am Institut für Angewandte Physik und Zentrum für Mikrostrukturforschung der Universität Hamburg. Seit 1998 Leitung des Kompetenzzentrums Nanoanalytik. Zahlreiche Auszeichnungen: Gaede-Preis 1992, Max Auwärter-Preis 1992, Karl Heinz Beckurts-Preis 1999, Mitglied der Deutschen Akademie der Naturforscher Leopoldina 2000, Philip Morris-Preis 2003 u.a.

1. Auflage April 2003

Alle Rechte vorbehalten

C B. G. Teubner / GWW Fachverlage GmbH, Wiesbaden 2003

Der B. G. Teubner Verlag ist ein Unternehmen der Fachverlagsgruppe BertelsmannSpringer. www.teubner.de

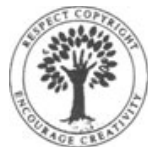

Das Werk einschließlich aller seiner Teile ist urheberrechtlich geschützt. Jede Verwertung außerhalb der engen Grenzen des Urheberrechtsgesetzes ist ohne Zustimmung des Verlags unzulässig und strafbar. Das gilt insbesondere für Vervielfältigungen, Übersetzungen, Mikroverfilmungen und die Einspeicherung und Verarbeitung in elektronischen Systemen.

Die Wiedergabe von Gebrauchsnamen, Handelsnamen, Warenbezeichnungen usw. in diesem Werk berechtigt auch ohne besondere Kennzeichnung nicht zu der Annahme, dass solche Namen im Sinne der Warenzeichen- und Markenschutz-Gesetzgebung als frei zu betrachten wären und daher von jedermann benutzt werden dürften.

Umschlaggestaltung: Ulrike Weigel, www.CorporateDesignGroup.de

Gedruckt auf säurefreiem und chlorfrei gebleichtem Papier. 


\section{Vorwort}

Wohl in keinem Kurs des Curriculums der Physik sind die Lernziele so vielfältig wie in der "Einführung in die Struktur der Materie". Viele physikalische Ergebnisse und Methoden müssen übersichtsmäßig behandelt werden. Die physikalischen Grundlagen sollen klar herausgestellt werden. Und schließlich soll ein Einblick in das experimentelle und theoretische Handwerkszeug vermittelt werden. Bei der Breite des Stoffs werden öfters qualitative Vorstellungen zur Erläuterung herangezogen - wie dies auch in der Forschung oft der erste Schritt ist. Aber auch die quantitative Beschreibung darf nicht vergessen werden - sie ist schließlich das Ziel der Physik.

Was wird vorausgesetzt? Neben Physik I und II (Mechanik, Elektrizitätslehre und Optik, Wärmelehre und statistische Physik) ist die Atomphysik einschliesslich der Grundlagen der Quantenmechanik (Physik III) eine unerläßliche Voraussetzung.

Die Ziele des Buchs sind:

1. Beschreibung der grundlegenden Phänomene der Struktur der Materie,

2. Einführung in die Meßmethoden (wirklich experimentieren lernt man allerdings nur im Praktikum und in der Diplomarbeit),

3. anschauliches Verständnis der Theorie (nicht der Rechentechniken),

4. Hinweise auf einige Anwendungen der Physik in anderen Gebieten.

Die Forschung ist vernetzt. Deshalb wird den Kapiteln der Kern- und Teilchenphysik zunächst ein Überblick an Hand der geschichtlichen Entwicklung vorangestellt. Dadurch wird die Vernetzung der Gebiete sowie von Experimenten, experimenteller Technik und theoretischen Ideen und Rechnungen klar. Der Weg der Forschung, der zu den wesentlichen Entdeckungen (oft auf Umwegen) geführt hat, wird deutlich. Danach kann der Stoff systematisch behandelt werden. Es wird möglich, im Text auf spätere Kapitel zu verweisen, wo ein Begriff, der vorher gebraucht wurde, ausführlich begründet wird. In den Kapiteln der Molekül- und Festkörperphysik erfolgt eine Beschränkung auf die wesentlichsten Grundlagen, die als Voraussetzung für weiterführende Spezialvorlesungen benötigt werden. 
An wen wendet sich das Buch? Die Autoren denken in erster Linie an Physikstudenten vor oder kurz nach dem Vordiplom bzw. an Studenten im Bachelor-Studiengang, die sich einen Überblick über die verschiedenen Gebiete der Physik verschaffen wollen, bevor sie speziellere Vorlesungen zu den einzelnen Gebieten im Rahmen des Aufbaustudiums bzw. MasterStudiengangs hören. Diese spezielleren Vorlesungen werden sich wiederum an den Forschungsschwerpunkten der jeweiligen Hochschule ausrichten. Der Stoffumfang dieses Buchs orientiert sich an einer vierstündigen einsemestrigen Vorlesung mit zwei Übungsstunden pro Woche. Aber auch für Gymnasiallehrer und Physiker in der Industrie wird das vorliegende Buch als Nachschlagewerk nützlich sein.

Welches Niveau strebt das Buch an? Der Ausgangspunkt sind experimentelle Beobachtungen. Diese werden theoretisch beschrieben. Formeln verdeutlichen einen physikalischen Sachverhalt am besten. Oft können nur die physikalischen Vorstellungen, die zu Grunde liegen, erläutert werden. Dann wird die Formel angegeben und benutzt. Eine Ableitung von Formeln kann nur in wenigen Fällen erfolgen.

Hinweise für den Leser

1. Lernziele: Jedem Abschnitt wurde ein Kasten mit "Lernzielen" vorangestellt. Darin wird der Inhalt des Abschnitts zusammengefaßt.

2. Aufgaben: Die Aufgaben haben unterschiedliche Schwierigkeitsgrade. Teilweise dienen sie dem quantitativen Verständnis der Formeln, teilweise soll die Auslegung von Experimenten überlegt werden. Wieder andere sind Denkaufgaben. Nach Möglichkeit wurde zu jedem wichtigen Themenfeld eine Aufgabe beigefügt.

Der Rahmen einer Vorlesung und eines Buchs ist beschränkt. Weiterführende Literatur ist angegeben. Ferner sind die genauen Zitate in einem gesonderten Literaturverzeichnis zu finden.

Dank: Johann Bienlein dankt zuerst seinem Doktorvater, Prof. Dr. Rudolf Fleischmann (Erlangen, 1903-2002), der das rechte Maß zwischen Anleitung und Freiheit der jungen Studenten einzuhalten wußte. Ferner gilt sein Dank Freunden und Kollegen auf den verschiedenen Etappen des Berufswegs. Hervorheben möchte er Prof. Dr. Horst Wegener (Erlangen) und Prof. Dr. Peter Stähelin (Hamburg). Nicht zu vergessen sind die Studenten, die im Gespräch Ideen und Formulierungen geschärft haben. Ein ganz besonderer Dank gilt seinem Kollgen Dr. Dieter Haidt für die kritische Durchsicht des Manuskripts und wertvollen Anregungen.

Roland Wiesendanger möchte mit diesem Buch seinem Physiklehrer Bernd Kretschmer danken, der es in hervorragender Weise verstanden hat, In- 
teresse und Begeisterung für die Physik zu wecken. Höchster Dank gilt schließlich Prof. Dr. Gerd Binnig und Dr. Heinrich Rohrer, die mit Ihrer Erfindung des Rastertunnelmikroskops ein neues Zeitalter der Festkörperphysik begründet haben.

Das Buch hätte nicht erstellt werden können ohne die tatkräftige Mithil$f e$ von Frau Angela Balbach und Frau Ursula Rehder sowie den Herren Dr. Mathias Getzlaff, Stefan Kuck, Boris Prinz und André Rothkirch. Dem Teubner-Verlag danken wir für die ausgezeichnete Zusammenarbeit. Schließlich möchten wir unseren Familien danken:

Frau Melanie Bienlein-Röllin und den Kindern Barbara, Regula und Martin, sowie Frau Silvia Wiesendanger und den Kindern Pascal und Dominik. Ihre große Geduld und stetige Ermunterung haben dieses Buch erst ermöglicht.

Hamburg, im November 2002

Johann Bienlein

Roland Wiesendanger 


\section{Inhalt}

$1 \quad$ Grundlagen der Struktur der Materie 1

1.1 Was heißt "Struktur der Materie"? . . . . . . . . . . . . 1

1.2 Grundlagen der Quantenmechanik . . . . . . . . . . . 3

1.2.1 Welle-Teilchen Dualismus . . . . . . . . . . . . . 3

1.2.2 Die Schrödinger-Gleichung . . . . . . . . . . . 6

1.2.3 Bahndrehimpuls und Spin . . . . . . . . . . . . . 8

1.2 .4 Das Pauli-Prinzip . . . . . . . . . . . . . . 10

1.2 .5 Aufgaben . . . . . . . . . . . . . 10

1.3 Beispiele für Anwendungen der Quantenmechanik . . . . . 11

1.3.1 Der Tunneleffekt . . . . . . . . . . . . . . . . . 11

1.3.2 Gebundener Zustand (Teilchen im Potential) . . . . . . . . 12

1.3 .3 Aufgaben ................... 15

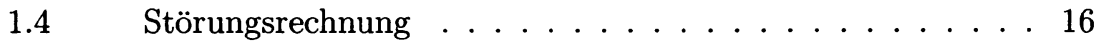

1.4 .1 Aufgaben . . . . . . . . . . . . . 17

$1.5 \quad$ Das Dipolmatrixelement . . . . . . . . . . . . 18

1.5 .1 Aufgaben .................... 19

$1.6 \quad$ Streuprozesse . . . . . . . . . . . . . . . 20

1.6.1 Grundbegriffe . . . . . . . . . . . . . . 20

1.6.2 Streuung und Reaktionen von Teilchen . . . . . . . . 22

1.6.3 Die Rutherford'sche Streuformel . . . . . . . . . . . . 23

1.6.4 Quantenmechanische Beschreibung der Streuung . . . . . 26

1.6.5 Aufgaben . . . . . . . . . . . . . . 27

$1.7 \quad$ Kinematik . . . . . . . . . . . . . . 28

$1.7 .1 \quad$ Überblick . . . . . . . . . . . . . . . . . . . 28

1.7.2 Relativistische Koordinatentransformation . . . . . . . 28

1.7.3 Transformation vom Labor- ins Schwerpunktsystem . . . . 30

1.7.4 Relativistisch invariante Größen . . . . . . . . . . . 32

1.7.5 Kinematischer Fit an Meßergebnisse . . . . . . . . . 33

$1.7 .6 \quad$ Aufgaben . . . . . . . . . . . . . . 34 
1.8 Ausblick auf die Struktur der Materie . . . . . . . . . 35

$2 \quad$ Konzepte und Instrumente der Kern- und Teilchenphysik 36

$2.1 \quad$ Konzepte der Kernphysik . . . . . . . . . . . . 36

2.1.1 Von der Entdeckung der Radioaktivität bis zum Neutron . 37

2.1.2 Aufklärung der Kernstruktur und der Kernreaktionen . . . 38

$2.2 \quad$ Konzepte der Teilchenphysik . . . . . . . . . . . . . . 43

2.2.1 Die Vorläufer und ihre Resultate . . . . . . . . . . . . . 44

2.2.2 Teilchenphysik der Hadronen . . . . . . . . . . . . 47

2.2.3 Teilchenphysik der Quarks (seit 1974) . . . . . . . . 50

2.3 Experimentelle Hilfsmittel: Teilchenbeschleuniger . . . . . . 54

2.3.1 Warum Teilchenbeschleuniger? . . . . . . . . . . . 54

2.3.2 Prinzip der Teilchenbeschleuniger ... . . . . . . 55

2.3.3 Aufbau der wichtigsten Beschleunigerarten . . . . . . . 56

2.3.4 Beschleunigerphysik .............. . 6 60

2.3 .5 Speicherringe . . . . . . . . . . . . . 63

2.3.6 Beispiel eines Beschleunigerkomplexes . . . . . . . 6 66

2.3.7 Aufgaben . . . . . . . . . . . . . . . 69

$2.4 \quad$ Experimentelle Hilfsmittel: Teilchendetektoren . . . . . . 70

2.4.1 Prinzip des Teilchennachweises . . . . . . . . . . 70

2.4.2 Ionisation der Materie . . . . . . . . . . . . . . 71

2.4.3 Teilchennachweis . . . . . . . . . . . . 78

2.4 .4 Statistik . . . . . . . . . . . . . 83

2.4 .5 Große Detektoren . . . . . . . . . . . . . . 86

2.4.6 Datenerfassung und -verarbeitung . . . . . . . . . 89

2.4.7 Strahlengefährdung und Strahlenschutz . . . . . . . . . 92

2.4 .8 Aufgaben . . . . . . . . . . . . . . . . 102

$3 \quad$ Kernphysik 104

3.1 Radioaktivität . . . . . . . . . . . . . . . . . 104

3.1 .1 Aufgaben .................... 106

$3.2 \quad$ Kerne und Kernbausteine . . . . . . . . . . . . . 107

3.2.1 Die Entdeckung des Atomkerns . . . . . . . . . . . 107

3.2 .2 Isotopie . . . . . . . . . . . . . . . . . . . 108

3.2 .3 Die Kernbausteine . . . . . . . . . . . . . . . 109

3.2 .4 Aufgaben . . . . . . . . . . . . . . . . 109

3.3 Systematik des Grundzustandes der Kerne . . . . . . . . . 110

3.3.1 Die Nuklidkarte . . . . . . . . . . . . . . . . . 110

3.3.2 Massendefekt und Bindungsenergie . . . . . . . . 112 
3.3.3 Erklärung der Bindungsenergie im Tröpfchenmodell . . . . 113

3.3.4 Stabile und instabile Kerne . . . . . . . . . . . . . . 114

$3.3 .5 \quad$ Der Kernspin . . . . . . . . . . . . . . . . 115

3.3.6 Die magnetischen Momente der Kerne . . . . . . . . . . 116

3.3 .7 Kernradien . . . . . . . . . . . . . . . 118

3.3 .8 Aufgaben . . . . . . . . . . . . . . . 120

$3.4 \quad$ Kernkräfte . . . . . . . . . . . . . . 121

3.4.1 Die Kernkraft als neues Phänomen . . . . . . . . . . . 121

3.4 Das Deuteron . . . . . . . . . . . . . . . . . . 122

3.4.3 Nukleon-Nukleon-Streuung . . . . . . . . . . . . 125

3.4.4 Mesonentheorie der Kernkräfte . . . . . . . . . . . 127

3.4 .5 Aufgaben . . . . . . . . . . . . . . 128

$3.5 \quad$ Kernreaktionen . . . . . . . . . . . . . . . . 129

3.5.1 Begriffe und Definitionen . . . . . . . . . . . 129

3.5.2 Erhaltungssätze . . . . . . . . . . . . . . . 130

3.5.3 Messung von Kernreaktionen . . . . . . . . . . . . 131

3.5.4 Theoretische Beschreibung von Kernreaktionen . . . . . . 132

3.5.5 Mechanismus von Kernreaktionen . . . . . . . . . . . 135

3.5 .6 Aufgaben . . . . . . . . . . . . . . . 139

3.6 Kernspektroskopie und Kernmodelle . . . . . . . . . . . 140

3.6.1 Experimente zur Kernspektroskopie . . . . . . . . . . . 140

3.6.2 Grundlagen der Kernstruktur . . . . . . . . . . . . . . . 144

3.6.3 Das Schalenmodell . . . . . . . . . . . . . . . . . 145

3.6 .4 Das Kollektivmodell . . . . . . . . . . . . . . . . 150

3.6.5 Das statistische Modell . . . . . . . . . . . . . 151

3.6 .6 Kernmaterie . . . . . . . . . . . . . . . 152

3.6 .7 Aufgaben ..................... 153

$3.7 \quad$ Neutronenphysik . . . . . . . . . . . . . 155

3.7 .1 Neutronenquellen . . . . . . . . . . . . . 155

3.7.2 Abbremsung von Neutronen . . . . . . . . . . . 157

3.7.3 Kernreaktionen von Neutronen . . . . . . . . . . . 158

3.7.4 Kernspaltung . . . . . . . . . . . . . . 159

3.7.5 Neutronendosimetrie und -abschirmung . . . . . . . . 161

3.7.6 Quantenzustände der Neutronen im Gravitationsfeld . . . . 162

3.7 .7 Aufgaben . . . . . . . . . . . . . . 164

$3.8 \quad$ Betazerfall . . . . . . . . . . . . . . 167

3.8 .1 Was ist der Betazerfall? . . . . . . . . . . . . 167

3.8.2 Die Messung des Elektronenspektrums . . . . . . . . . 168

3.8 .3 Das Neutrino . . . . . . . . . . . . . . 170 
3.8.4 Die Fermi-Theorie des Betazerfalls . . . . . . . . . . . 170

3.8.5 Positronenemission, Elektroneneinfang,

Rückstoßexperimente . . . . . . . . . . . . 173

3.8.6 Kern- $\beta$-Zerfälle . . . . . . . . . . . . . . 173

3.8.7 Was ist Paritätsverletzung? . . . . . . . . . . . . . 175

3.8.8 Messung der Elektronenpolarisation . . . . . . . . . 176

3.8.9 Die Form der Wechselwirkung des $\beta$-Zerfalls . . . . . . 178

3.8.10 Das Goldhaber-Experiment . . . . . . . . . . . . . 178

3.8 .11 Aufgaben . . . . . . . . . . . . . . . . 180

$3.9 \quad$ Neue Trends der Kernphysik . . . . . . . . . . . . . . 181

3.9.1 Kerne mit seltsamen Bausteinen ${ }^{8} \ldots \ldots \ldots$. . . . . . 181

3.9.2 Schwerionenphysik . . . . . . . . . . . . . . . . 182

3.9.3 Transurane und die "Insel der Stabilität" . . . . . . . . . 183

3.9.4 Streuung hochenergetischer Elektronen an Kernen . . . . 186

3.9.5 Aufgaben . . . . . . . . . . . . . . . . 190

$3.10 \quad$ Beispiele für Anwendungen der Kernphysik . . . . . . . . 191

3.10.1 Meßtechnik . . . . . . . . . . . . . . . . . . . 192

3.10 .2 Anwendungen in der Medizin . . . . . . . . . . . . . 194

3.10 .3 Kernreaktoren . . . . . . . . . . . . . . . . . . 199

3.10 .4 Aufgaben . . . . . . . . . . . . . . 209

4 Teilchenphysik $\quad 211$

4.1 Quantenelektrodynamik . . . . . . . . . . . . 211

4.1.1 Was ist Quantenelektrodynamik? . . . . . . . . . . 212

4.1.2 Antiteilchen . . . . . . . . . . . . . . . . 213

4.1.3 Feynman-Graphen . . . . . . . . . . . . . . 216

4.1.4 Einige QED Prozesse . . . . . . . . . . . . . . 220

4.1 .5 Positronium . . . . . . . . . . . . . . . . . 224

4.1.6 Renormierung der QED . . . . . . . . . . . . . . 225

4.1.7 Gültigkeitsgrenzen der QED . . . . . . . . . . . . 227

4.1 .8 Aufgaben . . . . . . . . . . . . . 228

4.2 Hadronische Reaktionen . . . . . . . . . . . . . . 230

4.2.1 Die Entdeckung des Pions . . . . . . . . . . . . . 230

4.2.2 Die Entdeckung seltsamer Teilchen . . . . . . . . . . 232

4.2.3 Die Entdeckung der Antiprotonen $(\bar{p}) \ldots . . \ldots 233$

4.2.4 Der Spin der Hadronen . . . . . . . . . . . . . 234

4.2.5 Erhaltungssätze bei Teilchenreaktionen . . . . . . . . . 235

4.2.6 Die invariante Masse instabiler Teilchen . . . . . . . . . 237

4.2.7 Wirkungsquerschnitte bei hohen Energien . . . . . . . . 239

4.2 .8 Aufgaben . . . . . . . . . . . . . . . 245 
$4.3 \quad$ Hadronenspektroskopie und Quarks . . . . . . . . . . . . 247

4.3.1 Multipletts von Hadronen . . . . . . . . . . . . . . . . 247

4.3.2 Die Quarks . . . . . . . . . . . . . . . . . 250

4.3.3 Aufbau der Hadronen aus Quarks . . . . . . . . . . 251

4.3.4 Hadronische Zerfälle der Resonanzen . . . . . . . . . . . . 253

4.3.5 Suche nach freien Quarks . . . . . . . . . . . . . . 254

4.3 .6 Aufgaben . . . . . . . . . . . . . . . 254

4.4 Lepton-induzierte Reaktionen . . . . . . . . . . . . 256

4.4 .1 Überblick . . . . . . . . . . . . . . . . . 256

4.4.2 Tief-inelastische Elektronstreuung . . . . . . . . . 257

4.4.3 Entdeckung der Partonen . . . . . . . . . . . . . 263

4.4.4 Aufgaben . . . . . . . . . . . . . . . . 264

4.5 Quantenchromodynamik . . . . . . . . . . . 267

4.5.1 Experimentelle Grundlagen der QCD . . . . . . . . 268

4.5.2 Theorie der QCD . . . . . . . . . . . . . . . 268

4.5.3 Experimentelle Bestätigung der QCD . . . . . . . . . 271

4.5.4 Das Hadronenspektrum im Lichte der QCD . . . . . . . . 276

4.5.5 Die Struktur der Nukleonen . . . . . . . . . . . . . . 277

4.5.6 Das Quark-Gluon-Plasma . . . . . . . . . . . . . . 282

4.6 Schwere Quarks und Hadronen . . . . . . . . . . . . 290

4.6.1 Entdeckung der Charme- und Bottom-Quarks . . . . . . 290

4.6.2 Quarkonia . . . . . . . . . . . . . . . . 296

4.6.3 Hadronen mit Charme- und Bottom-Flavor . . . . . . . . . 301

4.6.4 Aufgaben . . . . . . . . . . . . . . . . . . . 302

4.7 Schwache Wechselwirkung und CP-Verletzung . . . . . 304

4.7.1 Überblick . . . . . . . . . . . . . . . . . . . . . 305

$4.7 .2 \quad$ Der $\mu$-Zerfall . . . . . . . . . . . . . . 306

4.7 .3 Die $\pi$-Zerfälle . . . . . . . . . . . . . . . . 307

4.7.4 Zerfälle seltsamer Teilchen . . . . . . . . . . . 308

4.7.5 Das $\tau$-Lepton . . . . . . . . . . . . . . . . . . 309

4.7.6 Die Neutrino-Experimente . . . . . . . . . . . . . 311

4.7.7 Zerfälle der Charme- und Bottom-Hadronen . . . . . . . . 317

4.7.8 Die CKM-Matrix . . . . . . . . . . . . . . . . . . . . . 319

4.7.9 $K^{0}$-Zerfälle und CP-Verletzung . . . . . . . . . . . 321

4.7 .10 Aufgaben . . . . . . . . . . . . . . . 328

4.8 Elektroschwache Wechselwirkung . . . . . . . . . . 331

4.8.1 Divergenz der Fermi-Theorie . . . . . . . . . . . . . . 331

4.8.2 Die Entdeckung der neutralen schwachen Wechselwirkung . 333

4.8.3 Vereinheitlichung zur elektroschwachen Wechselwirkung . . 334 
4.8.4 Experimentelle Bestätigung der elektroschwachen Theorie . 337

4.8.5 Messungen mit $Z^{0}$-Zerfällen . . . . . . . . . . . . . . . 341

4.8.6 Der Higgs-Mechanismus . . . . . . . . . . . . . . . . . . 342

4.8.7 Entdeckung des Top-Quarks . . . . . . . . . . . . 343

4.8.8 Neutrino-Oszillationen . . . . . . . . . . . . . . . . 346

4.8 .9 Aufgaben . . . . . . . . . . . . . . . . 349

$4.9 \quad$ Standardmodell und Ausblick . . . . . . . . . . . . . . 351

4.9.1 Zusammenfassung: Teilchen und Wechselwirkungen . . . . 352

4.9 .2 Eichtheorien . . . . . . . . . . . . . . 353

4.9.3 Fragen an das Standardmodell . . . . . . . . . . . . . 354

4.9.4 Vorschläge für eine Erweiterung der Standardmodells . . . 355

4.9.5 Grundfrage der Teilchenphysik . . . . . . . . . . . . 356

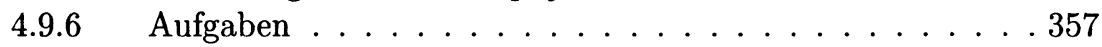

4.10 Der Wissenschaftsbetrieb der Teilchenphysik . . . . . . 358

$4.11 \quad$ Kosmische Strahlung . . . . . . . . . . . . . 360

4.11.1 Die Entdeckung der kosmischen Strahlung . . . . . . . . . . 360

4.11.2 Die kosmische Strahlung auf Meereshöhe . . . . . . . . . 361

4.11.3 Die primäre kosmische Strahlung . . . . . . . . . . . . 363

4.11.4 Astroteilchenphysik . . . . . . . . . . . . . . . 368

4.11 .5 Aufgaben . . . . . . . . . . . . . . . 372

4.12 Astrophysik: Neutrinos von der Sonne . . . . . . . . . . 374

4.12.1 Woher bezieht die Sonne die Energie? . . . . . . . . . . . 374

4.12.2 Grundbegriffe der Astrophysik . . . . . . . . . . . . 375

4.12.3 Energie-Erzeugung durch Kernfusion . . . . . . . . . . . . 379

4.12.4 Neutrino-Emission bei der solaren Kernfusion . . . . . . . . 381

4.12.5 Beobachtung der solaren Neutrinos . . . . . . . . . . . . 383

4.12.6 Messung der $\nu$-Oszillationen und des totalen $\nu$-Flußes durch SNO . . . . . . . . . . . . . . . . 388

4.12.7 Eine Lehre für die Wissenschaftler . . . . . . . . . . . . . . 391

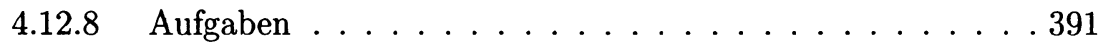

$5 \quad$ Molekülphysik $\quad 392$

$5.1 \quad$ Einführung . . . . . . . . . . . . . . . . 392

5.2 Die einfachsten Moleküle: $\mathrm{H}_{2}^{+}$und $\mathrm{H}_{2} \ldots \ldots . \ldots 394$

$5.2 .1 \mathrm{H}_{2}^{+}$- Molekülion . . . . . . . . . . . . . . 394

$5.2 .2 \quad \mathrm{Das}_{2}-$ Molekül $\ldots \ldots . \ldots . \ldots . \ldots 99$

$5.3 \quad$ Verschiedene Näherungsverfahren . . . . . . . . . . . . . 399

5.3.1 Molekülorbital-Näherung . . . . . . . . . . . . . 399 
5.3.2 Heitler-London-Näherung . . . . . . . . . . . . . 401

5.3.3 Vergleich zwischen Molekülorbital-Näherung und

Heitler-London-Näherung . . . . . . . . . . . . . . . . . 404

5.3 .4 Näherung der Valenzbindung . . . . . . . . . . . 405

$5.4 \quad$ Hybridisierung . . . . . . . . . . . . . 406

$5.5 \quad$ Arten der chemischen Bindung . . . . . . . . . . . . . 409

5.5 .1 Kovalente Bindung . . . . . . . . . . . . . . . . . . . . . . . . . . . . .

5.5 .2 Ionische Bindung . . . . . . . . . . . . . . . . 411

5.5 .3 Metallische Bindung . . . . . . . . . . . . 411

5.5.4 Wasserstoffbrücken-Bindung . . . . . . . . . . 411

5.5 .5 Van-der-Waals-Bindung . . . . . . . . . . . . 411

5.6 Empirische Wechselwirkungspotentiale . . . . . . . . 413

5.7 Molekulare Anregungen . . . . . . . . . . . . . 415

5.7 .1 Elektronische Anregung . . . . . . . . . . . . . . 415

5.7 .2 Vibrations-/ Schwingungsanregung . . . . . . . . . . 416

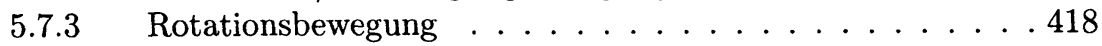

5.7 .4 Molekülspektrum . . . . . . . . . . . . . . . . . . . . . . . . . .

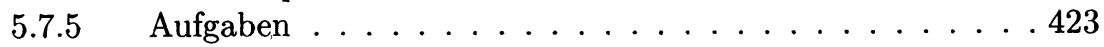

$6 \quad$ Festkörperphysik $\quad 425$

$6.1 \quad$ Einführung . . . . . . . . . . . . . . 425

6.2 Chemische Bindung in Festkörpern . . . . . . . . . 428

$6.3 \quad$ Festkörperstruktur . . . . . . . . . . . . . . 432

6.3.1 Beschreibung von Kristallstrukturen (Kristallographie) . . 432

6.3.2 Aufgaben . . . . . . . . . . . . . . . . . . 442

6.3.3 Experimentelle Bestimmung von Kristallstrukturen . . . .443

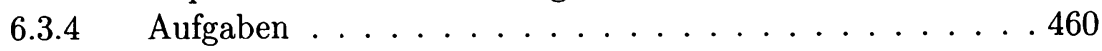

6.4 Einteilung der Festkörperphysik . . . . . . . . . 462

6.5 Gitterdynamik . . . . . . . . . . . . . . . 464

6.5.1 Gitterschwingungen in einer eindimensionalen periodischen

Struktur . . . . . . . . . . . . . . . 467

6.5.2 Gitterschwingungen in einer dreidimensionalen periodischen Struktur . . . . . . . . . . . . . 471

6.5.3 Wechselwirkungsfreies Phononengas . . . . . . . . . 474

6.5.4 Phononenzustandsdichte . . . . . . . . . . . . 477

6.5.5 Experimentelle Bestimmung der Phononendispersion und der Phononenzustandsdichte . . . . . . . . . . . 481

$6.5 .6 \quad$ Spinwellen . . . . . . . . . . . . . . 485 
6.5.7 Aufgaben . . . . . . . . . . . . . . 486

6.6 Makrosk. Festkörpereigenschaften im thermodyn. Gleich-

$6.6 .1 \quad$ Einteilung . . . . . . . . . . . . . . . . 488

6.6.2 Thermische Eigenschaften des Kristallgitters . . . . . . . 489

6.6.3 Aufgaben . . . . . . . . . . . . . . . . . . 493

6.7 Makrosk. Festkörpereigenschaften außerhalb des thermodyn. Gleichgew. . . . . . . . . . . . . . . . . 494

6.7.1 Einteilung . . . . . . . . . . . . . . . . . . . . . 494

6.7.2 Wärmeleitung des Kristallgitters . . . . . . . . . . 494

6.8 Wechselwirkungsfreies Elektronengas . . . . . . . . . . 496

6.8.1 Grundzustand des Elektronengases für $T=0 \ldots 499$

6.8.2 Elektronengas bei endlicher Temperatur $(T>0) \ldots 501$

6.8.3 Thermische Eigenschaften des Elektronengases . . . . . . . 503

6.8.4 Elektrische Transporteigenschaften des Elektronengases . . 505

6.8.5 Magnetische Eigenschaften des Elektronengases . . . . . . . 507

6.8.6 Aufgaben . . . . . . . . . . . . . . 511

6.9 Elektronen im periodischen Potential . . . . . . . . . 513

6.9.1 Blochtheorem . . . . . . . . . . . . . . . . . 514

6.9.2 Bandstruktur . . . . . . . . . . . . . . 515

6.9.3 Elektronenzustandsdichte . . . . . . . . . . . . 518

6.9 .4 Halbleiter . . . . . . . . . . . . . . . . . . 522

6.9 .5 Aufgaben . . . . . . . . . . . . . . . . . . . . . .

$6.10 \quad$ Supraleitung . . . . . . . . . . . . . . 527

$7 \quad$ Weiterführende Literatur $\quad 533$

$\begin{array}{ll}\text { Literaturverzeichnis } & \mathbf{5 3 5}\end{array}$

A Einheiten, Konstanten und Formeln $\quad 538$

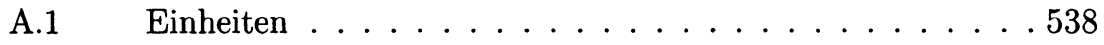

A.2 Wichtige Konstanten und Umrechnungsfaktoren . . . . . 539

A.3 Präfixe für Vielfache und Teile von Einheiten . . . . . . . 540

A.4 Abkürzungen . . . . . . . . . . . . . . 540

$\begin{array}{ll}\text { Sachverzeichnis } & 546\end{array}$ 